\title{
Rastreamento do câncer do colo do útero na cidade de Belém, Estado do Pará, Brasil
}

Screening for cervical cancer in the city of Belém, State of Pará, Brazil

Cribado de cáncer de cuello uterino en la ciudad de Belém, Estado de Pará, Brasil

José Lima Pereira Filho ORCID: https://orcid.org/0000-0001-7163-3831 Universidade Federal do Maranhão, Brasil

E-mail: jlp.filho@outlook.com

Árlon Wendel Marinho da Silva Araújo ORCID: https://orcid.org/0000-0002-9281-2676 Universidade Federal do Maranhão, Brasil E-mail: arlonwendellg3@gmail.com

Emanuele Fernanda Lima Ribeiro ORCID: https://orcid.org/0000-0002-6217-487X

Universidade Federal do Maranhão, Brasil

E-mail:emanuele.fernanda@discente.ufma.br

Rafael Arouche

ORCID: https://orcid.org/0000-0002-2375-6244 Universidade Federal do Maranhão, Brasil E-mail: rafaelarouche12@gmail.com

Pedro Henrique Pereira Lopes

ORCID: https://orcid.org/0000-0002-4910-0809 Universidade Federal do Maranhão, Brasil E-mail: pedro.henrique.pereira.lopes.3@gmail.com

Samuel dos Santos Soares Buna

ORCID: https://orcid.org/0000-0002-2411-5123 Universidade Federal do Maranhão, Brasil

E-mail: samuelbuna@hotmail.com

Breno Facundes Bonfim

ORCID: https://orcid.org/0000-0001-7107-0960 Universidade Federal do Maranhão, Brasil

E-mail: brenobonfim1@gmail.com

Rayssa Katherynne Lopes Anunciação ORCID: https://orcid.org/0000-0002-9572-2692 Universidade Federal do Maranhão, Brasil E-mail: rayssa.lopez@outlook.com

Joyce Caroline Gomes da Costa

ORCID: https://orcid.org/0000-0002-0671-347X

Universidade Federal do Maranhão, Brasil

E-mail: joyce.caroline@discente.ufma.br

Wesley Flory do Nascimento Costa

ORCID: https://orcid.org/0000-0002-4005-4471

Universidade Federal do Maranhão, Brasil

E-mail: wesley.flory@discente.ufma.br

George Luiz Ribeiro de Araujo

ORCID: https://orcid.org/0000-0001-5616-8396

Faculdade Estácio São Luís, Brasil

E-mail: george.luiz@yahoo.com.br

Tarliene dos Santos

ORCID: https://orcid.org/0000-0002-0038-0088 Faculdade Pitágoras, Brasil

E-mail: julianatarlienesantos24@gmail.com

Ana Zélia Silva

ORCID: https://orcid.org/0000-0001-6565-2868

Universidade Federal do Maranhão, Brasil E-mail: anazelia.silva@yahoo.com.br

Ahirlan Silva de Castro

ORCID: https://orcid.org/0000-0003-1954-289X

Universidade Federal do Maranhão, Brasil

E-mail:ahirlan.castro@ufma.br

Iracelle Carvalho Abreu

ORCID: https://orcid.org/0000-0002-3286-6816

Universidade Federal do Maranhão, Brasil

E-mail: iracellec@yahoo.com.br 


\author{
Selma do Nascimento Silva \\ ORCID: https://orcid.org/0000-0002-2896-4990 \\ Universidade Federal do Maranhão, Brasil \\ E-mail: selma.silva@ufma.br
}

\begin{abstract}
Resumo
O câncer cervical ou de colo do útero (CCU) é um tumor maligno feminino considerado um grave problema de saúde pública e que apresenta altos índices de morbimortalidade em todo o mundo. Este trabalho objetivou avaliar o perfil epidemiológico e citopatológico dos cânceres de colo de útero que foram diagnosticados na cidade de Belém no período de 2016 a 2020. Foi desenvolvido um estudo epidemiológico de natureza descritiva, com abordagem quantitativa e retrospectiva com coleta de dados referentes à cidade de Belém, no Estado do Pará, Brasil. Estes dados foram coletados a partir do Sistema de Informação do Câncer (SISCAN), base de dados disponibilizada pelo Departamento de Informática do Sistema Único de Saúde (DATASUS) entre os meses de Outubro e Novembro de 2021. Foram registrados 206.614 exames citopatológicos no período de estudo, sendo 2019 o ano que apresentou o maior número de exames realizados $(32,23 \%)$. A faixa etária com o maior número de exames compreendia entre 30 a 34 anos, correspondendo a 12,87\%. Quanto à adequabilidade, $89,55 \%$ das amostras classificaram-se como satisfatórias. O principal motivo para a realização do exame foi o de Rastreamento $(97,84 \%)$. O epitélio escamoso foi a amostra citopatológica mais representada (89,69\%). Dentre as alterações celulares benignas, a inflamação apresentou maior quantidade de registros $(82,83 \%)$ e, entre as anormalidades celulares mais recorrentes, estão as lesões intraepiteliais de baixo grau (2,98\%). Este estudo aborda a necessidade da realização periódica de exames preventivos, uma vez que é uma doença passível de tratamento quando identificada em estágio inicial.
\end{abstract}

Palavras-chave: HPV; Teste de Papanicolau; Perfil epidemiológico e citopatológico; Prevenção; Belém.

\begin{abstract}
Cervical or cervical cancer (CCU) is a female malignant tumor considered a serious public health problem and has high morbidity and mortality rates worldwide. This study aimed to evaluate the epidemiological and cytopathological profile of cervical cancers that were diagnosed in the city of Belém in the period from 2016 to 2020 . An epidemiological study of a descriptive nature, with a quantitative and retrospective approach, was developed with data collection for the city of Belém, in the State of Pará, Brazil. These data were collected from the Cancer Information System (SISCAN), a database made available by the Information Technology Department of the Unified Health System (DATASUS) between the months of October and November 2021. A total of 206,614 cytopathological tests were registered during the study period, with 2019 being the year with the highest number of tests performed $(32,23 \%)$. The age group with the highest number of exams was between 30 and 34 years old, corresponding to $12,87 \%$. As for the suitability, $89,55 \%$ of the samples were classified as satisfactory. The main reason for performing the exam was for tracking $(97,84 \%)$. The squamous epithelium was the most represented cytopathological sample $(89,69 \%)$. Among the benign cellular alterations, inflammation had the highest number of records $(82,83 \%)$ and, among the most recurrent cellular abnormalities, are low-grade intraepithelial lesions (2.98\%). This study addresses the need for periodic preventive examinations, since it is a treatable disease when identified at an early stage.
\end{abstract}

Keywords: HPV; Pap test; Epidemiological and cytopathological profile; Prevention; Belém.

\title{
Resumen
}

El cáncer de cuello uterino o de cuello uterino (UCC) es un tumor maligno femenino considerado un problema grave de salud pública y tiene altas tasas de morbilidad y mortalidad en todo el mundo. Este estudio tuvo como objetivo evaluar el perfil epidemiológico y citopatológico de los cánceres cervicouterinos que fueron diagnosticados en la ciudad de Belém en el período de 2016 a 2020. Se desarrolló un estudio epidemiológico de carácter descriptivo, con enfoque cuantitativo y retrospectivo, con recolección de datos para la ciudad de Belém, en el Estado de Pará, Brasil. Estos datos fueron recolectados del Sistema de Información del Cáncer (SISCAN), una base de datos puesta a disposición por el Departamento de Tecnología de la Información del Sistema Único de Salud (DATASUS) entre los meses de octubre y noviembre de 2021. Durante el período de estudio se registraron un total de 206.614 pruebas citopatológicas, siendo 2019 el año con mayor número de pruebas realizadas (32,23\%). El grupo de edad con mayor número de exámenes fue el de 30 a 34 años, correspondiente al 12,87\%. En cuanto a la idoneidad, el 89,55\% de las muestras se clasificaron como satisfactorias. La principal razón de el examen se realizó a través del Screening $(97,84 \%)$. El epitelio escamoso fue la muestra citopatológica más representada $(89,69 \%)$. Entre las alteraciones celulares benignas, la inflamación tuvo el mayor número de registros $(82,83 \%)$ y, entre las anomalías celulares más recurrentes, se encuentran las lesiones intraepiteliales de bajo grado (2,98\%). Este estudio aborda la necesidad de exámenes preventivos periódicos, ya que es una enfermedad tratable cuando se identifica en una etapa temprana.

Palabras clave: VPH; Prueba de Papanicolaou; Perfil epidemiológico y citopatológico; Prevención; Belém. 


\section{Introdução}

O câncer do colo do útero (CCU) é uma das principais causas de morte por câncer entre as mulheres e o quarto tumor maligno feminino mais incidente em todo o mundo (Pereira \& Vieira, 2021), sendo que a infecção persistente com genótipos carcinogênicos do papilomavírus humano (HPV) é a causa de quase todos os cânceres cervicais (Pedersen et al., 2018).

O número de casos novos de câncer do colo do útero esperados para o Brasil, para cada ano do triênio 2020-2022, será de 16.590, com um risco estimado de 15,43 casos a cada 100 mil mulheres. No Estado do Pará, o CCU é o segundo mais comum entre as mulheres e apresentou uma estimativa de 780 novos casos em 2020, e uma taxa de 18,24 casos para cada 100.000 mil mulheres (INCA, 2019).

O HPV é um vírus que pode ser transmitido sexualmente, e o DNA do HPV de alto risco está presente em 99,7\% das amostras de câncer cervical. Dentro de 12 a 24 meses de exposição ao vírus, 90\% das infecções por HPV são eliminadas ou se tornam inativas. No entanto, as infecções por tipos de HPV de alto risco persistentes, podem aumentar o risco de progressão para CCU (Chan et al., 2019). Mesmo sendo uma condição necessária, a infecção pelo HPV não apresenta uma causa suficiente para o surgimento desse câncer, sendo que outros fatores interferem na progressão desse tumor (Thuler et al., 2012).

Vários estudos têm avaliado fatores de risco para infecção genital pelo HPV. Esses fatores de risco incluem o número de parceiros sexuais, início precoce da atividade sexual, novo parceiro sexual, tabagismo, uso prolongado de contraceptivos orais, histórico de infecções sexualmente transmissíveis (IST's), imunossupressão, baixo nível sócio-econômico e predisposição genética (Pancera \& Santos, 2018; Kuiava \& Chielle, 2019). Ressalta-se que todos esses fatores são mutáveis, consequentemente, são passíveis de medidas de intervenção e, portanto, os profissionais e órgãos de saúde têm importante papel orientador na prevenção dessa condição (Kuiava \& Chielle, 2019). Entretanto, apesar da possibilidade de prevenção, observa-se ainda que, em cerca de $50 \%$ dos casos, a doença é diagnosticada em estágio avançados, tornando o seu tratamento mais agressivo e diminuindo as possibilidades de cura (Thuler et al., 2012).

O CCU tem uma distribuição bimodal por idade, com a maioria dos casos ocorrendo entre mulheres na faixa dos 30 e 40 anos, idade em que as mulheres costumam constituir família e garantir a viabilidade financeira de suas famílias e comunidades. Além do risco de morte, o câncer do colo do útero está associado ao aumento da morbidade, incluindo sangramento, dor e insuficiência renal, que são difíceis de tratar, especialmente em comunidades com pouco acesso a serviços de saúde (Chan et al., 2019).

Programas de rastreamento organizados com o objetivo de detectar e tratar os pré-cânceres do colo do útero antes que progridam para o câncer, têm contribuído para reduzir a carga do câncer cervical (Pedersen et al., 2018). No Brasil a prevenção do câncer cervical é classificada em primária e secundária. A primária compõe-se de medidas que visam diminuir o risco de contágio pelo HPV, sendo a vacinação contra esse vírus e o uso regular de preservativos os seus principais constituintes. A secundária, por sua vez, refere-se às estratégias de rastreamento e diagnóstico precoce que é fundamentada na realização periódica do exame citopatológico, iniciando-se aos 25 anos, devendo seguir até os 64 anos (Vaz et al., 2020).

O conhecimento da epidemiologia deste tumor é um ponto importante e fundamental para o planejamento de ações objetivas para a redução das taxas de morbimortalidade por esta doença (Kuiava \& Chielle, 2019).

Em virtude da grande prevalência e da elevada morbimortalidade associada a essa doença, esse estudo objetivou avaliar o perfil epidemiológico e citopatológico do câncer do colo do útero diagnosticados no período de 2016 a 2020 na cidade de Belém, Estado do Pará. Tendo isso em vista, realizou-se pesquisas em diferentes bases de dados on-line com o intuito de analisar artigos que abordem o grau de conhecimento do público-alvo sobre a realização do exame preventivo de Papanicolau e, dessa forma, ser possível delinear um perfil de prevenção do CCU. 


\section{Metodologia}

Trata-se de um estudo epidemiológico de natureza descritiva e com abordagem quantitativa e retrospectiva (Pereira et al., 2018). Os dados coletados são referentes a cidade de Belém, Estado do Pará, Brasil. As informações foram coletadas a partir do Sistema de Informação do Câncer (SISCAN), base de dados disponibilizada pelo Departamento de Informática do Sistema Único de Saúde (DATASUS) entre os meses de Outubro e Novembro de 2021 (Pereira Filho et al., 2021). O DATASUS é um órgão pertencente à Secretaria Executiva do Ministério da Saúde que é responsável por coletar, processar e disseminar informações sobre os principais indicadores de saúde do Brasil (Ferraz, 2009).

O SISCAN permite que as unidades de saúde informatizadas e com acesso a internet sejam usuários do sistema para fazer a solicitação de exames, visualizar os resultados e acompanhar as mulheres com exames alterados, monitorando o seguimento. Somado a isso, esse sistema possibilita registrar a suspeita, confirmação diagnóstica e informações das condutas diagnósticas e terapêuticas relativas aos exames positivo e/ou alterados (Alves, 2020).

O alvo deste estudo foi a população feminina de Belém entre os 15 a 69 anos de idade. Os dados foram coletados entre o período de 2016 a 2020. Selecionou-se como parâmetros epidemiológicos e citopatológicos a faixa etária, ano resultado, citologia anterior, adequabilidade da amostra, motivo do exame, epitélios e alterações celulares do exame. Todos os dados coletados foram processados no Programa Microsoft Excel 2016, onde estes foram tabelados em planilhas (Pereira Filho et al., 2021).

Com o intuito de gerar maior embasamento científico e discussão sucinta dos resultados, buscou-se pesquisar artigos na literatura científica para compreender o grau de conhecimento das mulheres sobre o exame preventivo de Papanicolau. Realizaram-se pesquisas nas bases de dados PUBMED (Medical Literature Analysis and Retrieval System Online), Lilacs (Literatura Latino-americana e do Caribe em Ciências da Saúde) e periódicos CAPES, aplicando-se os seguintes descritores em português, espanhol e inglês: HPV; Câncer do Colo de Útero; Mortalidade Feminina; Epidemiologia; HPV; Cáncer de cuello uterino; Mortalidad femenina; Epidemiología; Cervical Cancer; Female Mortality; Epidemiology.

Tendo em vista a Resolução n 466/12 do Conselho Nacional de Saúde (CNS), este projeto de pesquisa não foi submetido à apreciação ética do Conselho de Ética em Pesquisa (CEP) em seres humanos em virtude do fato de utilizar dados de domínio público em site da internet e, dessa forma, sendo dispensado da aplicação de termo de consentimento livre e esclarecido (TCLE), uma vez que os dados foram coletados no cadastro do SISCAN (Freitas; Guerra \& Brito, 2020).

\section{Resultados e Discussão}

O meio de rastreio do CCU e de suas lesões precursoras é feito através do exame citopatológico de Papanicolau. A finalidade essencial do rastreio é identificar e tratar de forma prévia as lesões precursoras antes do seu progresso para a doença invasiva. Assim, com o alcance da população-alvo de, no mínimo, $80 \%$ e alcance à confirmação diagnóstica e ao tratamento adequado, é capaz de reduzir em até $90 \%$ a incidência do câncer cervical invasivo (Caldeira \& Justino, 2020).

Mediante a observação dos dados presentes na Tabela 1, é possível quantificar que no período de 2016 a 2020, foram registrados no SISCAN o equivalente a 260.614 exames citopatológicos de mulheres residentes no município de Belém, Estado do Pará, Brasil. O ano de 2019 apresentou a maior quantidade de exames registrados equivalente a 84.013 (32,23\%), seguido do ano de 2018 com 68.595 (26,32\%) e 2017 com 36.439 (13,98\%) exames.

Apesar do aumento no número de exames realizados nos últimos anos verifica-se que no ano de 2020 houve uma redução substancial no número de exames realizados. Essa redução está relacionada diretamente com a pandemia pelo novo coronavírus, uma vez que o estabelecimento da pandemia da COVID-19 traz diversas repercussões para a área da saúde. Além de todas as implicações que a infecção pelo Sars-Cov-2 traz em si, com o aumento no número de infectados faz-se necessário repensar a busca pelos serviços de saúde a fim de conter a contaminação. Dentro deste contexto, diversos órgãos de saúde se 
manifestaram orientando que consultas, exames e cirurgias que não tenham carácter de urgência fossem adiadas (Militão et al., 2021).

Tabela 1. Número de exames citopatológicos realizados no período de 2016 a 2020 em Belém, Pará, Brasil.

\begin{tabular}{ccc}
\hline ANO & NÚMERO DE EXAMES & $\mathbf{( \% )}$ \\
\hline 2016 & 28.702 & 11.01 \\
2017 & 36.439 & 13.98 \\
2018 & 68.595 & 26.32 \\
2019 & 84.013 & 32.23 \\
2020 & 42.865 & 16.44 \\
\hline TOTAL & 260.614 & 100 \\
\hline
\end{tabular}

Fonte: Ministério da Saúde - Sistema de Informação do Câncer (SISCAN).

O aumento crescente no número de novos exames registrados ao longo dos anos pode estar associado a campanhas de educação em saúde que estimulam estas mulheres a realizarem o exame preventivo de Papanicolau. Este exame pode ser considerado um dos maiores avanços da medicina oncológica dos últimos anos, já que embora seja um exame indolor, simples e rápido, por meio de um esfregaço do colo do útero, possui grande impacto no desfecho da doença em questão, sendo protagonista nos temas relacionados a câncer de colo de útero e HPV (Machado et al., 2017).

No Brasil, as recomendações do Ministério da Saúde indicam os 25 anos como idade para início do exame de mulheres que já iniciaram a atividade sexual, devendo ser realizado até os 64 anos e, após esse período, podendo ser interrompido quando tiverem dois exames negativos consecutivos nos últimos cinco anos (Silva et al., 2018).

Com a finalidade de melhorar a cobertura do programa de prevenção do CCU, foram estipulados indicadores com a finalidade de avaliar o alcance de metas e as estratégias do programa de enfrentamento do CCU em mulheres dentro da população alvo de 25 a 64 anos de idade (Souza et al., 2018). De acordo com Souza e colaboradores (2018), 80\% dos exames citopatológicos devem ser realizados nessa faixa etária. Neste estudo, 82,63\% das mulheres apresentavam idade entre 25 a 64 anos (Tabela 2).

De acordo com a Tabela 2, verifica-se o número de exames citopatológicos realizados por faixa etária no período de estudo. Observa-se que a faixa etária entre 35 a 39 anos apresentou a maior quantidade de exames realizados (13,25\%), seguido dos 30 a 34 anos $(12,87 \%)$ e 25 a 29 anos $(11,67 \%)$.

Nota-se que o exame também é realizado com mulheres mais jovens entre a faixa etária de 15 a 24 anos de idade, correspondendo um total de 37.499 (14,38\%). Sabe-se que as mulheres jovens estão iniciando a vida sexual cada vez mais cedo, em muitos casos ainda imaturas, despreparadas e desorientadas, sendo fator de risco para o desenvolvimento do câncer de colo do útero, visto que foram expostas muito jovens a pessoas com HPV e a outros vírus (Kuiava \& Chielle, 2019). 
Tabela 2. Número de exames citopatológicos realizados por faixa etária no período de 2016 a 2020 em Belém, Pará, Brasil.

\begin{tabular}{|c|c|c|c|c|c|c|c|}
\hline \multirow{3}{*}{ FAIXA ETÁRIA } & \multicolumn{5}{|c|}{ ANO DO EXAME } & \multirow{3}{*}{ TOTAL } & \multirow{3}{*}{$(\%)$} \\
\hline & 2016 & 2017 & 2018 & 2019 & 2020 & & \\
\hline & $\mathbf{n}$ & $\mathbf{n}$ & n & $\mathbf{n}$ & $\mathbf{n}$ & & \\
\hline 15 a 19 & 1.358 & 1.750 & 3.070 & 3.510 & 1.778 & 11.466 & 4.40 \\
\hline 20 a 24 & 2.931 & 3.765 & 6.998 & 8.282 & 4.057 & 26.033 & 9.98 \\
\hline 25 a 29 & 3.820 & 4.457 & 7.955 & 9.756 & 4.427 & 30.415 & 11.67 \\
\hline 30 a 34 & 4.300 & 4.888 & 8.717 & 10.549 & 5.094 & 33.548 & 12.87 \\
\hline 35 a 39 & 4.034 & 5.047 & 9.085 & 10.993 & 5.373 & 34.532 & 13.25 \\
\hline 40 a 44 & 3.367 & 4.277 & 8.141 & 10.079 & 5.222 & 31.086 & 11.93 \\
\hline 45 a 49 & 2.924 & 3.909 & 7.499 & 9.068 & 4.890 & 28.290 & 10.86 \\
\hline 50 a 54 & 2.417 & 3.269 & 6.529 & 8.307 & 4.467 & 24.989 & 9.59 \\
\hline 55 a 59 & 1.797 & 2.443 & 5.015 & 6.215 & 3.517 & 18.987 & 7.29 \\
\hline 60 a 64 & 1.146 & 1.660 & 3.510 & 4.645 & 2.516 & 13.477 & 5.17 \\
\hline \multirow[t]{2}{*}{65 a 69} & 608 & 974 & 2.076 & 2.609 & 1.524 & 7.791 & 2.99 \\
\hline & & & & & & 260.614 & 100 \\
\hline
\end{tabular}

Fonte: Ministério da Saúde - Sistema de Informação do Câncer (SISCAN).

Devido ao seu fácil diagnóstico em estágios precoces por meio da técnica de Papanicolau, houve uma importante redução da morbimortalidade por essa patologia ao longo das últimas décadas, nos países que implementaram massivamente programas de rastreamento e tratamento (Tsuchiya et al., 2017). No Brasil, entretanto, apesar do avanço nas políticas públicas para o controle do CCU, a maioria dos diagnósticos ainda é feita em fases avançadas da doença.

Entre os principais motivos para a não realização do exame preventivo está o desconhecimento, que faz com que, em muitas vezes, só haja procura e realização do exame apenas quando há sinais e sintomas. A maior parte das mulheres procura atendimento ginecológico, incluindo realização da citologia preventiva, somente nos casos onde existe sintomatologia, fato que comprova e reafirma o desconhecimento das mesmas sobre a importância do exame preventivo em questão (Castro, 2010).

Mediante a Tabela 3, verifica-se que a quantidade de mulheres que afirmaram ter realizado exame citopatológico anterior à consulta $(78,63 \%)$ é superior àquelas que afirmaram desconhecer $(6,18 \%)$ ou não terem realizado o exame $(13,33 \%)$.

Tabela 3. Citologia anterior realizada no período de 2016 a 2020 em Belém, Pará, Brasil.

\begin{tabular}{|c|c|c|c|c|c|c|c|}
\hline \multirow{3}{*}{ CITOLOGIA ANTERIOR } & \multicolumn{5}{|c|}{ ANO DO EXAME } & \multirow{3}{*}{ TOTAL } & \multirow{3}{*}{$(\%)$} \\
\hline & 2016 & 2017 & 2018 & 2019 & 2020 & & \\
\hline & $\mathbf{n}$ & $\mathbf{n}$ & $\mathbf{n}$ & $\mathbf{n}$ & $\mathbf{n}$ & & \\
\hline Sim & 23.244 & 28.803 & 52.858 & 65.673 & 34.340 & 204.918 & 78.63 \\
\hline Não & 3.749 & 4.792 & 9.812 & 10.863 & 5.513 & 34.729 & 13.33 \\
\hline Não Sabe & 1.517 & 2.321 & 4.067 & 5.870 & 2.338 & 16.113 & 6.18 \\
\hline \multirow[t]{2}{*}{ Sem Informação } & 192 & 513 & 1.843 & 1.637 & 669 & 4.854 & 1.86 \\
\hline & & & & & & 260.614 & 100 \\
\hline
\end{tabular}

Fonte: Ministério da Saúde - Sistema de Informação do Câncer (SISCAN).

De acordo com estudos sobre os determinantes da cobertura do exame de rastreio do CCU no Brasil, a não realização do exame associa-se à baixa escolaridade, ao baixo nível socioeconômico, à baixa renda familiar, ao viver sem companheiro, à cor parda, ao uso de contraceptivo oral, à vergonha ou ao medo em relação ao exame, ao fato de não ter realizado consulta médica no ano anterior à pesquisa e a dificuldade de acesso à assistência médica (Fonseca, 2010).

O desconhecimento das mulheres sobre o câncer do colo do útero e sua relação com o HPV reforça que, quanto menos se conhece sobre este vírus, menos se tem a capacidade de prevenir corretamente esse agente oncogênico (Sousa \& Miranda, 2018). 
A partir da Tabela 4, observam-se os principais motivos para a realização dos exames citopatológicos entre as mulheres residentes de Belém.

Tabela 4. Motivo para a realização dos exames citopatológicos no período de 2016 a 2020, no município de Belém, Pará, Brasil.

\begin{tabular}{|c|c|c|c|c|c|c|c|}
\hline \multirow{3}{*}{ MOTIVO DO EXAME } & \multicolumn{5}{|c|}{ ANO DO EXAME } & \multirow{3}{*}{ TOTAL } & \multirow{3}{*}{$(\%)$} \\
\hline & 2016 & 2017 & 2018 & 2019 & 2020 & & \\
\hline & $\mathbf{n}$ & $\mathbf{n}$ & $\mathbf{n}$ & $\mathbf{n}$ & $\mathbf{n}$ & & \\
\hline Rastreamento & 27.740 & 35.290 & 67.372 & 82.779 & 41.824 & 255.005 & 97.84 \\
\hline Repetição (exame alterado: ASC-US/Baixo grau) & 337 & 422 & 396 & 578 & 421 & 2.154 & 0.83 \\
\hline \multirow[t]{2}{*}{ Seguimento } & 625 & 727 & 827 & 656 & 620 & 3.455 & 1.33 \\
\hline & & & & & & 260.614 & 100 \\
\hline
\end{tabular}

Fonte: Ministério da Saúde - Sistema de Informação do Câncer (SISCAN).

Com base nos dados supracitados, o rastreamento (97,84\%) correspondeu ao principal motivo para realização do exame, seguido de "repetição" (0,83\%) e "seguimento" (1,33\%). Em um estudo realizado por Corrêa et al., (2012), a maioria das mulheres relatou a procura espontânea pelo exame $(66,2 \%)$, seguida das que realizaram o exame por recomendação médica $(23,5 \%)$ e das que referiram à presença de queixas ginecológicas como a principal razão para a busca do exame (10,3\%). Ainda de acordo com esse estudo, a maioria das mulheres (96,8\%) recebeu informação sobre o Papanicolaou, sendo a Unidade Básica de Saúde (UBS) mencionada como a principal fonte de informação sobre o exame, seguido de outros meios (rádio/TV, escola, revista/jornal, amigos/parentes, igreja). Dessa forma, é possível notar que o auxílio dos profissionais de saúde no que tange a educação em saúde é essencial para o estímulo da realização periódica dos exames preventivos do CCU pelas mulheres.

Através da observação dos dados presente na Tabela 5 é possível analisar a distribuição dos exames realizados quanto à adequabilidade da amostra exames citopatológicos. Verifica-se que 89,55\% das amostras foram caracterizadas como “satisfatórias", seguido das "rejeitadas" $(9,82 \%)$ e "insatisfatórias" $(0,63 \%)$.

Designa-se satisfatória, a amostra que apresente células em quantidade suficiente, bem distribuídas, fixadas e coradas, de tal modo que sua visualização permita uma conclusão diagnóstica (Freitas et al., 2020).

Tabela 5. Distribuição dos exames realizados, quanto à adequabilidade da amostra, segundo o período analisado de 2016 a 2020, no município de Belém, Pará, Brasil.

\begin{tabular}{|c|c|c|c|c|c|c|c|}
\hline \multirow{3}{*}{ ADEQUABILIDADE } & \multicolumn{5}{|c|}{ ANO DO EXAME } & \multirow{3}{*}{ TOTAL } & \multirow{3}{*}{$(\%)$} \\
\hline & 2016 & 2017 & 2018 & 2019 & 2020 & & \\
\hline & $\mathbf{n}$ & $\mathbf{n}$ & $\mathbf{n}$ & $\mathbf{n}$ & $\mathbf{n}$ & & \\
\hline Rejeitada & 3.759 & 5.628 & 5.064 & 6.240 & 4.897 & 25.588 & 9.82 \\
\hline Satisfatória & 24.676 & 30.540 & 63.018 & 77.348 & 37.805 & 233.387 & 89.55 \\
\hline \multirow[t]{2}{*}{ Insatisfatória } & 267 & 271 & 513 & 425 & 163 & 1.639 & 0.63 \\
\hline & & & & & & 260.614 & 100 \\
\hline
\end{tabular}

Fonte: Ministério da Saúde - Sistema de Informação do Câncer (SISCAN).

Souza e colaboradores (2018) definem que o percentual de amostras insatisfatórias no total de exames realizados em um determinado local e período tem que ser inferior a 5\% do total de exames. Estes indicadores estão diretamente relacionados com a coleta do material e informam o percentual de amostras consideradas inadequadas, sendo estas insuficientes para o diagnóstico, necessitando de repetição do exame. Através da análise da Tabela 5, constata-se que o percentual de amostras insatisfatórias no município de Belém está dentro dos padrões preconizados, correspondendo a 0,63\% do total de exames. 
A adequabilidade da amostra e a representatividade celular apontam para a qualidade do procedimento realizado pelos profissionais de saúde. O despreparo teórico-prático do profissional resulta na baixa qualidade da coleta. Por esse motivo, é importante capacitar, atualizar e qualificar os profissionais envolvidos com o objetivo de aumentar o nível de satisfatoriedade das lâminas e representação celular (Rodrigues et al., 2016). Através dos resultados encontrados neste estudo, torna-se preocupante o alto percentual de amostras rejeitas $(9,82 \%)$, o que evidencia o despreparo dos profissionais que atuam na realização dos exames citopatológicos do colo uterino.

A qualidade diagnóstica dos exames citopatológicos e histopatológicos do colo do útero são fundamentais para que as ações de controle do câncer alcancem o sucesso esperado. Para tanto, se faz necessário reduzir o percentual de exames falsonegativos, falso-positivos e insatisfatórios (Claro et al., 2021).

Segundo Claro e colaboradores (2021), o ideal para a realização do exame citopatológico é que o esfregaço contenha a representação dos epitélios escamoso, glandular e/ou metaplásico. De acordo com a Tabela 6, verifica-se a presença dos principais epitélios representados na amostra citopatológica da população estudada. O epitélio escamoso foi o mais representado nas amostras citopatológicas (57\%), seguido pelo glandular (88\%) e metaplásico (22\%).

Tabela 6. Epitélios representados na amostra citopatológica da população estudada no período de 2016 a 2020 em Belém, Pará, Brasil.

\begin{tabular}{cccccccc}
\hline \multirow{3}{*}{ EPITÉLIOS } & \multicolumn{8}{c}{ ANO DO EXAME } & & \multirow{2}{*}{ TOTAL } & \multirow{2}{*}{$\boldsymbol{\%})$} \\
\cline { 2 - 6 } & $\mathbf{2 0 1 6}$ & $\mathbf{2 0 1 7}$ & $\mathbf{2 0 1 8}$ & $\mathbf{2 0 1 9}$ & $\mathbf{2 0 2 0}$ & & \\
\cline { 2 - 6 } & $\mathbf{n}$ & $\mathbf{n}$ & $\mathbf{n}$ & $\mathbf{n}$ & $\mathbf{n}$ & & \\
\hline Escamoso & 24.990 & 30.585 & 62.994 & 77.362 & 37.820 & 233.751 & 89.69 \\
\hline Glandular & 10.302 & 16.155 & 36.979 & 45.312 & 21.698 & 130.446 & 50.05 \\
\hline Metaplásico & 2.324 & 2.930 & 6.143 & 11.041 & 6.990 & 29.428 & 11.29 \\
\hline
\end{tabular}

Fonte: Ministério da Saúde - Sistema de Informação do Câncer (SISCAN).

A qualificação profissional para o preparo da lâmina é condição essencial, pois além da realização de uma boa coleta, o esfregaço precisa ser feito de forma bem distribuída e adequadamente fixado para melhor visualização quando a lâmina for corada. O ideal para a realização do exame citopatológico é que o esfregaço contenha a representação dos epitélios escamoso, glandular e/ou metaplásico. Contudo, para ser considerado satisfatório para análise, poderá conter apenas células do epitélio escamoso (Claro et al., 2021).

Ao se tratar do contexto clínico, Silva e colaboradores (2013) afirmam que para a boa qualidade do exame, é desejável que a representação dos epitélios glandulares e/ou metaplásicos seja pelo menos igual ao escamoso, sendo que a sua ausência é considerada normal somente nas mulheres submetidas à histerectomia. No entanto, a soma destes epitélios (61\%) foi abaixo do desejado (Tabela 6), o que pode ser um indício da má qualidade da coleta e/ ou fixação do material, provavelmente por despreparo teórico-prático dos profissionais para sua realização.

Em conformidade com o trabalho realizado por Pereira Filho e colaboradores (2021) sobre o rastreamento do CCU em mulheres residentes da cidade de São Luís, Estado do Maranhão, as alterações celulares dos exames foram divididas em três categorias: alterações celulares benignas (inflamação, atrofia com inflamação, metaplasia escamosa imatura, radiação, reparação e outros), anormalidades em células escamosas (lesão intraepitelial de baixo e alto grau, carcinoma epidermoide invasor) e anormalidades em células glandulares (adenocarcinoma in situ e adenocarcinoma invasor).

Mediante a Tabela 7, observam-se principais alterações celulares dos exames citopatológicos dos exames realizados na população estudada. 
Tabela 7. Análise das principais alterações celulares dos exames citopatológicos da população estudada no período de 2016 a 2020 em Belém, Pará, Brasil.

\begin{tabular}{|c|c|c|c|c|c|c|c|}
\hline \multirow{3}{*}{ ALTERAÇÕES CELULARES } & \multicolumn{5}{|c|}{ ANO DO EXAME } & \multirow{3}{*}{ TOTAL } & \multirow{3}{*}{$\%$} \\
\hline & 2016 & 2017 & 2018 & 2019 & 2020 & & \\
\hline & $\mathbf{n}$ & $\mathbf{n}$ & $\mathbf{n}$ & $\mathbf{n}$ & $\mathbf{n}$ & & \\
\hline \multicolumn{8}{|l|}{ Alterações celulares benignas } \\
\hline Inflamação & 21.422 & 25.119 & 50.688 & 64.369 & 30.490 & 192.088 & 82.83 \\
\hline Atrofia com inflamação & 1.458 & 2.532 & 6.009 & 6.780 & 4.444 & 21.223 & 9.15 \\
\hline Metaplasia escamosa imatura & 591 & 872 & 941 & 861 & 657 & 3.922 & 1.69 \\
\hline Radiação & 0 & 1 & 12 & 8 & 6 & 27 & 0.01 \\
\hline Reparação & 12 & 3 & 15 & 38 & 32 & 100 & 0.04 \\
\hline Outros & 5 & 7 & 7 & 1 & 2 & 22 & 0.01 \\
\hline \multicolumn{8}{|l|}{ Anormalidades em células escamosas } \\
\hline ASC-US & 682 & 675 & 1.142 & 1.660 & 844 & 5.003 & 2.16 \\
\hline ASC-H & 85 & 87 & 152 & 314 & 173 & 811 & 0.35 \\
\hline Lesão intraepitelial de baixo grau & 715 & 931 & 1.806 & 2.305 & 1.143 & 6.900 & 2.98 \\
\hline Lesão intraepitelial de alto grau & 210 & 258 & 326 & 489 & 259 & 1.542 & 0.66 \\
\hline Carcinoma epidermoide invasor & 28 & 18 & 39 & 60 & 33 & 178 & 0.08 \\
\hline \multicolumn{8}{|l|}{ Anormalidades em células glandulares } \\
\hline Adenocarcinoma in situ & 4 & 5 & 26 & 21 & 6 & 62 & 0.03 \\
\hline Adenocarcinoma invasor & 3 & 4 & 5 & 7 & 2 & 21 & 0.01 \\
\hline
\end{tabular}

Abreviação: ASC-US/ASC-H: células escamosas atípicas de significado indeterminado possivelmente não neoplásica ou não se pode afastar lesão de alto grau. Fonte: Ministério da Saúde - Sistema de Informação do Câncer (SISCAN).

De acordo com a Tabela 7, a inflamação foi o achado mais frequente entre as alterações celulares benignas $(82,83 \%)$, seguido da atrofia com inflamação $(9,15 \%)$ e metaplasia escamosa imatura $(1,69 \%)$.

Dentre as atipias supracitadas, as lesões intraepiteliais de baixo grau foram as mais recorrentes (2,98\%), seguido das lesões intraepiteliais de alto grau (0,66\%), carcinoma epidermoide invasor $(0,08 \%)$ e dos adenocarcinomas in situ $(0,03 \%)$ e invasor (0,01\%). Segundo Oliveira e colaboradores (2020), existem duas categorias de carcinomas consideradas como principais: o carcinoma epidermoide e o adenocarcinoma. O primeiro acomete o epitélio escamoso e é tido como o mais incidente, o qual possui responsabilidade por cerca de $80 \%$ dos casos. Ainda segundo o estudo desses autores, houve um predomínio de lesões escamosas intraepiteliais de baixo grau que pode acontecer devido à progressão da lesão ao longo do tempo ou subdiagnóstico da citopatologia, especialmente em mulheres com idade menor que 30 anos.

Diante disso, para a redução da incidência e mortalidade do CCU é importante à detecção das lesões precursoras em mulheres assintomáticas, por meio do exame citopatológico do colo do útero, fundamental para a eficiência de um programa de rastreamento organizado. Seguindo um conjunto de ações programadas, com a população-alvo, faixa etária e a periodicidade entre os exames bem definidas já que a efetividade da detecção precoce de lesões precursoras por meio do exame de Papanicolau, associada ao tratamento em seus estádios iniciais, tem resultado em redução de até 90,0\% nas taxas de incidência de câncer cervical invasor (Oliveira et al., 2020).

No Brasil, os altos índices de incidência e mortalidade por CCU evidenciam a importância da elaboração e da implementação de políticas públicas na atenção básica, voltadas à atenção integral à saúde da mulher, garantindo ações relativas ao controle dessa doença. Apesar dos avanços na difusão de medidas preventivas, o CCU continua a ser um problema de saúde importante no país. Os gestores e profissionais de saúde devem ser responsáveis pela realização dessas ações e devem possibilitar a integralidade do cuidado, unindo as ações de detecção precoce com a garantia de acesso a procedimentos diagnósticos e terapêuticos em tempo oportuno e com qualidade. A atenção básica qualificada e organizada é fundamental para o controle desses tipos de cânceres (Tsuchiya et al., 2017). Quando se fala em CCU, investir em medidas preventivas é consequentemente reduzir as taxas de incidência e melhorar a qualidade de vida dessas mulheres (Silva et al., 2016).

Ainda de acordo com o supracitado, as ações educativas devem ocorrer sobremaneira na atenção básica, que está mais próxima do cotidiano das mulheres e as acompanha ao longo da sua vida. As abordagens educativas devem estar presentes no 
processo de trabalho das equipes, seja em momentos coletivos, como grupos, atividades do Programa de Saúde na Escola, outras abordagens grupais da equipe, seja em momentos individuais de consulta. É de suma importância à disseminação da necessidade dos exames e da sua periodicidade, bem como dos sinais de alerta que podem significar câncer (Silva et al., 2016).

\section{Considerações Finais}

Por meio deste estudo, foi possível verificar que o número de exames citopatológicos realizados pelas mulheres em Belém aumentou progressivamente de 2016 a 2019. No entanto, apresentou uma queda em 2020 devido à pandemia do novo coronavírus, pois com o aumento no número de infectados fez-se necessário repensar a busca pelos serviços de saúde a fim de conter a contaminação, reduzindo assim o número de exames citopatológicos.

O Brasil possui avanços nas políticas públicas de controle do CCU, porém, a maioria dos diagnósticos é feita em estágios avançados da doença. A técnica de Papanicolau permite a identificação da doença em estágio precoce e isso reduziria o número de casos no país. Entretanto, assim como evidenciado nesse estudo, sabe-se que o desconhecimento acerca desse exame preventivo é um dos fatores que influenciam na progressão da doença, uma vez que a pessoa realiza o exame apenas quando há manifestações clínicas de sinais e sintomas.

Este trabalho também aponta a importância de que o profissional de saúde esteja em constante capacitação na coleta de amostras para realização do exame, já que um grande percentual de amostras foi classificado como insatisfatória ou rejeitada. É importante também que o profissional de saúde esteja qualificado na promoção de campanhas educativas acerca do CCU e na importância de se realizar o teste de Papanicolau para fins de prevenção, uma vez que este câncer é uma patologia passível de cura quando identificada em estágios iniciais.

Diante do exposto, almeja-se, com este trabalho, instigar a comunidade acadêmica a dedicar pesquisas, capacitação profissional e educação em saúde nessa área, com fito de que os exames de Papanicolau sejam realizados periodicamente e de forma adequada para que se obtenham altos índices de prevenção e cura do CCU. Nesse ínterim, destaca-se a importância da pesquisa no processo de saúde-doença, já que essa será um meio de inovação e melhoramento na realização das técnicas e exames existentes, principalmente, relacionada à saúde pública no Brasil. Sugere-se que pesquisas futuras busquem aumentar as variáveis estudadas para que se possa conhecer de forma mais profunda todos os aspectos epidemiológicos envolvidos nesse tipo de câncer em suas diferentes localidades. Somado a isso, deve-se buscar relatar as ações em que os profissionais da área da saúde podem realizar com o intuito de expandir o conhecimento e incentivarem as mulheres a procurar o serviço de saúde com o intuito da realização periódica dos exames preventivos.

\section{Referências}

Alves, A. S. (2020). Câncer de mama: avaliação do rastreamento através de indicadores de processo no Siscan. (Tese de Doutorado). Acesso no acervo do Programa de Pós-Graduação Interinstitucional em Oncologia da Fundação Antônio Prudente em parceria com o Hospital de Câncer de Pernambuco.

Brasil, Ministério da Saúde (BR) (2020). Secretaria Nacional de Assistência à Saúde. Instituto Nacional do Câncer - INCA.

Caldeira, J. R. F. \& Justino, G. C. M. (2021). Prevalência do câncer de colo uterino em Manhuaçu-MG: Importância da prevenção da prevenção na saúde da mulher $e$ suas interferências. (Trabalho de Curso). http://www.pensaracademico.unifacig.edu.br/index.php/repositoriotcc/article/view/3192/2247.

Castro, L. F. Exame Papanicolau: o conhecimento das mulheres sobre o preventivo e a estratégia do PSF no combate ao câncer de colo de útero. (Trabalho de Conclusão de Curso, Universidade Federal de Minas Gerais). https://repositorio.ufmg.br/bitstream/1843/BUOS9CPG5K/1/monografia_let_cia_ferreira_castro.pdf.

Chan, C. K. (2019). Human Papillomavirus Infection and Cervical Cancer: Epidemiology, Screening, and Vaccination-Review of Current Perspectives. Journal of oncology, 1-12.

Claro, I. B. et al. (2021). Análise dos Motivos de Insatisfatoriedade dos Exames Histopatológicos do Colo do Útero no Sistema Único de Saúde, Brasil, 2014 a 2017. Revista Brasileira de Cancerologia, 67(3), e081299. 
Côrrea, D. A. D., Villela, W. V. \& Almeida, A. M. (2012). Desafios à organização de programa de rastreamento do câncer do colo do útero em Manaus-AM. Texto \& Contexto-Enfermagem, 21(2), 395-400.

Ferraz, L. H. V. C. (2009). O SUS, o DATASUS e a informação em saúde: uma proposta de gestão participativa. (Dissertação de Mestrado, Escola Nacional de Saúde Pública Sergio Arouca).

Fonseca, A. J. et al. (2010). Epidemiologia e impacto econômico do câncer de colo de útero no Estado de Roraima: a perspectiva do SUS. Revista Brasileira de Ginecologia e Obstetrícia, 32(8), 386-392.

Freitas, M. S., Guerra, G. T. R. \& Britto, M. H. R. M. (2020). Perfil epidemiológico do câncer do colo do útero diagnosticado entre 2016 a 2019 em Teresina, Estado do Piauí, Brasil. Research, Society and Development, 9(10), e5309108877-e5309108877.

Kuiava, V. A \& Chielle, E. O. (2019). Epidemiology of cervix cancer in Brazil (2005-2015): study of mortality and hospital intervention rates. Arch Biosci Heal, 1(1), 45-60.

Machado, H. S., Souza, M. C. \& Gonçalves, S. J. C. (2017). Câncer de colo de útero: Análise Epidemiológica e Citopatológica no município de Vassouras RJ. Revista Pró-UniverSUS, 8(1), 55-61.

Militão, B. V. P. et al. (2021). Repercussões da pandemia de Sars-Cov-2 na realização do exame de Papanicolau: um estudo epidemiológico. Revista Eletrônica Acervo Saúde, 13(9), p. e8869-e8869.

Oliveira, E. H. (2020). Análise da prevalência do câncer de colo uterino em mulheres do Estado do Piauí, Brasil. Research, Society and Development, 9(10), e2509108540-e2509108540.

Pancera, T. R. \& Santos, G. H. N. (2018). Epidemiologia Molecular da infecção pelo papilomavírus humano (HPV) e câncer cervical no Brasil: Revisão Integrativa. Revista de Patologia do Tocantins, 5(2), 78-83.

Pedersen, K. (2018). An overview of cervical cancer epidemiology and prevention in Scandinavia. Acta Obstetricia et Gynecologica Scandinavica, 97, 795807.

Pereira, A. S. et al. (2018). Metodologia da pesquisa científica. http://uniesp.edu.br/sites/_biblioteca/revistas/20170627112856.pdf.

Pereira, M. V. S. \& Vieira, R. S. L. (2021). Rastreamento do câncer de colo de útero em mulheres mineiras de 2012-2015. Bionorte, $10(2)$, 160-167.

Pereira Filho, J. L. et al. (2021). Câncer do colo do útero: Análise epidemiológica e citopatológica no Município de São Luís, Estado do Maranhão, Brasil. Research, Society and Development, 10(8), e33010817074-e33010817074.

Rodrigues, J. F. et al. (2016). Rastreamento do câncer do colo do útero na região ampliada oeste de Minas Gerais. Revista de Enfermagem do Centro-Oeste Mineiro, 6(2), 2156-2168.

Sousa, K. R. \& Miranda, M. A. L. (2018). Câncer do colo do útero: percepção das mulheres frente ao exame preventivo. Comunicação em Ciências da Saúde, 29(3), 183-190.

Silva, R. C. G. (2018). Perfil de mulheres com câncer de colo do útero atendidas para tratamento em centro de oncologia. Revista Brasileira de Saúde Materno Infantil, 18(4), 703-710.

Silva, A. M. et al. (2016). Perfil epidemiológico do câncer do colo do útero na Paraíba. Temas em saúde, 16(4), $180-197$.

Thuler, L. C. S., Bergmann, A. \& Casado, L. (2012) Perfil das pacientes com câncer do colo do útero no Brasil, 2000-2009: Estudo de base secundária. Câncer do colo do útero no Brasil. Revista Brasileira de Cancerologia, 58(3), 351-357.

Tsuchiya, C. T. et al. (2017). O câncer de colo do útero no Brasil: uma retrospectiva sobre as políticas públicas voltadas à saúde da mulher. Jornal Brasileiro de Economia da Saúde, 9(1), 137-147.

Vaz, D. W. N. et al. (2020). Avaliação Epidemiológica do câncer do colo do útero no Estado do Amazonas. Revista Amazônia Science \& Health, 8(3), 61-69.

Zhang, S. et al. (2020). Cervical cancer: Epidemiology, risk factors and screening. Chinese Journal of Cancer Research, 32(6), 720-728. 